\title{
Mouldy Sioud (ed): siRNA and miRNA Gene Silencing: from Bench to Bedside
}

\author{
Methods in Molecular Biology. The Humana Press, 487 pp, ISBN 978-1-6173-7908-6
}

\author{
Francesco Nicassio
}

Published online: 9 March 2012

(C) Springer Science+Business Media, LLC 2012

This is one of the most comprehensive and recent books about gene targeting through siRNA and miRNA, intended to provide readers with an updated compendium of current strategies and methods towards therapeutic applications of gene silencing.

It starts with topics related to the optimization of siRNAs and their performance in vivo, mentioning design guide rules and new methods useful for designing siRNA sequences (i.e., statistical and clustering techniques) as well as chemical modifications (i.e., LNA) to prevent unwanted effects or activation of innate immunity. Nevertheless, the main challenges in using siRNA in vivo are the delivery, tissue/cell specific targeting and monitoring of siRNA release and potency in vivo. Therefore, most chapters are dedicated to these issues, focusing on several methods for siRNA delivery in live animals, such as magnetofection or nanoparticles using peptide-receptor interaction or atelocollagen matrix, and imaging approaches to monitor in vivo siRNA release and its effects (bioluminescence, MRI, radiolabelling). The book also covers topics related to intriguing new technologies, such as the use of TransKingdom RNAi (tkRNAi, Chapter 7) and bacterial mediated delivery of siRNA in solid tumors (Chapter 8). Special attention is given to therapeutic application of gene silencing through miRNA and siRNA, mentioning relevant examples of therapeutic target genes already validated in vitro or in vivo (Chapters 11-16), including oncogenes and viral proteins. Indeed, the book ends by describing the use of a synthetic siRNA against $\mathrm{Bcr}-\mathrm{Abl}$ fusion transcript in a patient with leukemia.

While being very detailed in the issues concerning siRNAs and their application in vivo, only few chapters are dedicated to miRNAs, mainly describing the microRNA pathway and biogenesis, with few mentions on miRNA therapeutics.

The text is suited for researchers, teachers, students and biotech companies interested in siRNA- and miRNA-based approach to gene regulation, which could benefit of a recently updated and comprehensive compendium of methods and strategies envisioned to foster the development of RNAi technology from bench to bedside.

F. Nicassio

Department of Experimental Oncology at IEO, European

Institute of Oncology, Via Ripamonti 435, 20141 Milan, Italy

F. Nicassio $(\bowtie)$

Center for Genomic Science of IIT@SEMM, Istituto Italiano di

Tecnologia, IFOM-IEO Campus, Via Adamello 16, 20139

Milan, Italy

e-mail: francesco.nicassio@ifom-ieo-campus.it 